\title{
Method for Measuring Reflectance of Semiconductor Disk Laser Gain Element Under Optical Pump Excitation
}

\author{
Carl Borgentun, Jörgen Bengtsson, and Anders Larsson \\ Photonics Laboratory, Department of Microtechnology and Nanoscience (MC2), Chalmers University of Technology, Göteborg, Sweden \\ carl.borgentun@chalmers.se
}

\begin{abstract}
We present a new measurement method for measuring the spectral reflectance of a semiconductor disk laser gain element under optical pumping, providing valuable information on the spectral dependence of gain under close-to-normal operating conditions.

OCIS codes: (140.5960) Semiconductor lasers; (120.5700) Reflection
\end{abstract}

\section{Introduction}

The optically pumped semiconductor disk laser (OP-SDL), or vertical-external-cavity surface-emitting laser (VECSEL), is a laser capable of generating a high-power beam of almost diffraction-limited quality $[1,2]$. The properties of the spatial modes of the OP-SDL emission are controlled by the external cavity but the power and spectral properties are to a large extent governed by the semiconductor layer structure of the gain element. It is therefore of utmost importance to be able to design the gain element such that the performance characteristics fit the preferred ones. A standard way to experimentally test the design is to perform a spectral reflectance measurement of the gain element in the absence of optical pumping, i.e., to simply measure the reflected optical power at a known incident power for several data points within a wavelength interval [3]. This is a straight-forward and simple measurement method but the retrieved information is restricted to the case of a cold cavity and little or no information is retrieved about the actual performance of the gain element under pump excitation.

We here present a measurement method that, as compared to the standard reflectance measurement method, reveals information on the spectral and power properties of the gain element under optical excitation and enables a direct comparison with simulation results. This comparison is very useful in the iterative evolution of gain element design and particularly advantageous for more advanced designs such as those optimized for wide tuning $[4,5]$ or for short pulse generation [6], since the measurement conditions more closely resemble the operating conditions in the actual OP-SDL cavity. In these kinds of design, a certain spectral dependence of the gain is sought after to render the wanted behavior of the OP-SDL.

\section{Setup}

The setup, schematically shown in Fig. 1, probes the reflectance of the gain element by employing a tunable laser with narrow bandwidth (e.g., a Ti-sapphire laser). The gain element is simultaneously being optically pumped, and due to stimulated emission from the active region the reflectance can exceed unity, i.e., produce gain. Thus the setup is capable of measuring the gain spectrum of a gain element. For practical purposes the setup needs to be calibrated to account for fluctuations in the output power of the probe laser and absorption in optical components. Therefore the probe beam is split by a wedged beam-splitter, and part of the beam is reflected onto a photo-detector used as

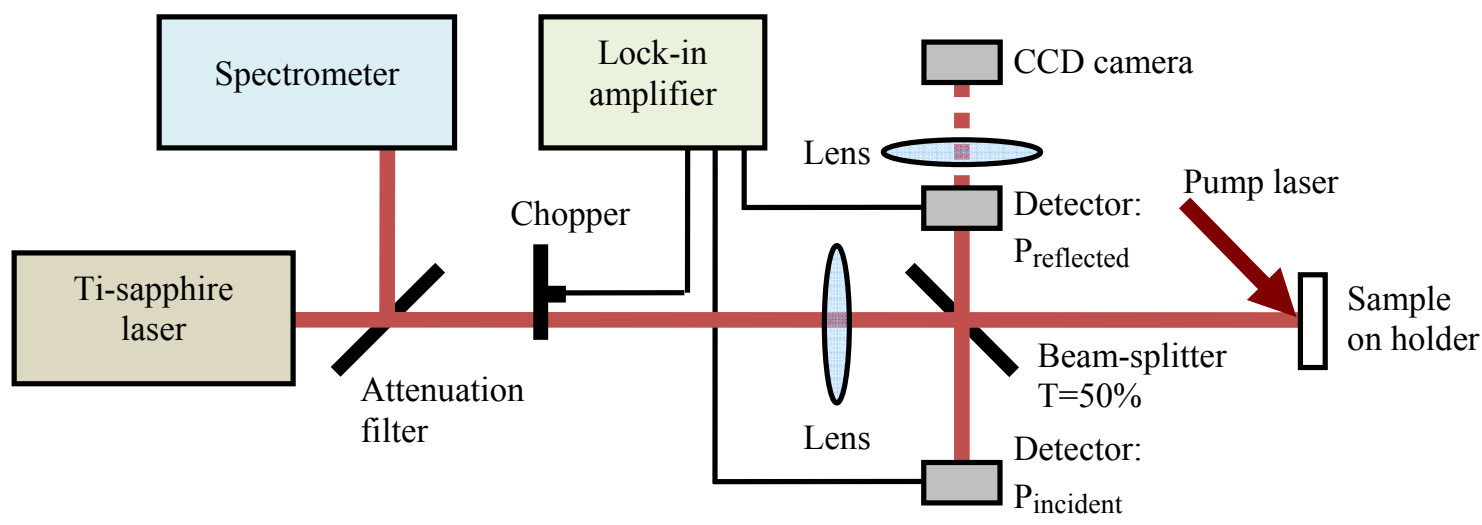

Fig. 1. Schematic overview of the measurement setup. When aligning the spots from the probe and pump lasers, the top detector is removed to let a lens and a CCD camera image the sample surface. 


\section{JTuI94.pdf}

reference of the incident power. The other part of the beam is incident on the optically pumped gain element, reflected back to the beam-splitter, where the light is reflected onto a second photo-detector. Due to uncertainties in the optical properties of the beam-splitter and the detectors, a calibration is necessary with a measurement on a sample with a known reflectance, e.g. a reflectance standard. The reflectance of the gain element can then be written:

$$
R(\lambda)=\frac{P_{\text {reflected }}(\lambda)}{P_{\text {incident }}(\lambda)} \cdot \frac{P_{\text {calibration,incident }}(\lambda)}{P_{\text {calibration,reflected }}(\lambda)} \cdot R_{\text {calibration }}(\lambda)
$$

The alignment of the probe and pump laser spots can be conveniently performed by removing the detector measuring the reflected power, thereby unblocking a lens that images the sample surface on a CCD camera. Naturally the pump spot has to completely cover the spot from the probe laser, and it might be necessary to insert a focusing lens that reduces the size of the probe spot on the sample so that the pump intensity can be sufficiently increased.

To prevent saturation of the detectors, an attenuation filter is inserted in the beam. This also functions as a beam-splitter as the reflected light is guided into a spectrometer for wavelength control.

\section{Preliminary results}

Initial measurements using the described setup have been performed on a gain element optimized for wide tuning (details on the structure can be found in [5]) and the calibration sample was a broadband dielectric mirror with a well-known reflectance. The preliminary measurement results, shown to the left in Fig. 2, reveal a significant response to pump excitation and agree well with the simulation results, shown to the right in Fig. 2. The measured pump intensities are approximate due to difficulties in accurately determining the pump spot size and the cause for differences is most likely approximations in the model and deviations in the fabricated epitaxial structure from the prescribed one.
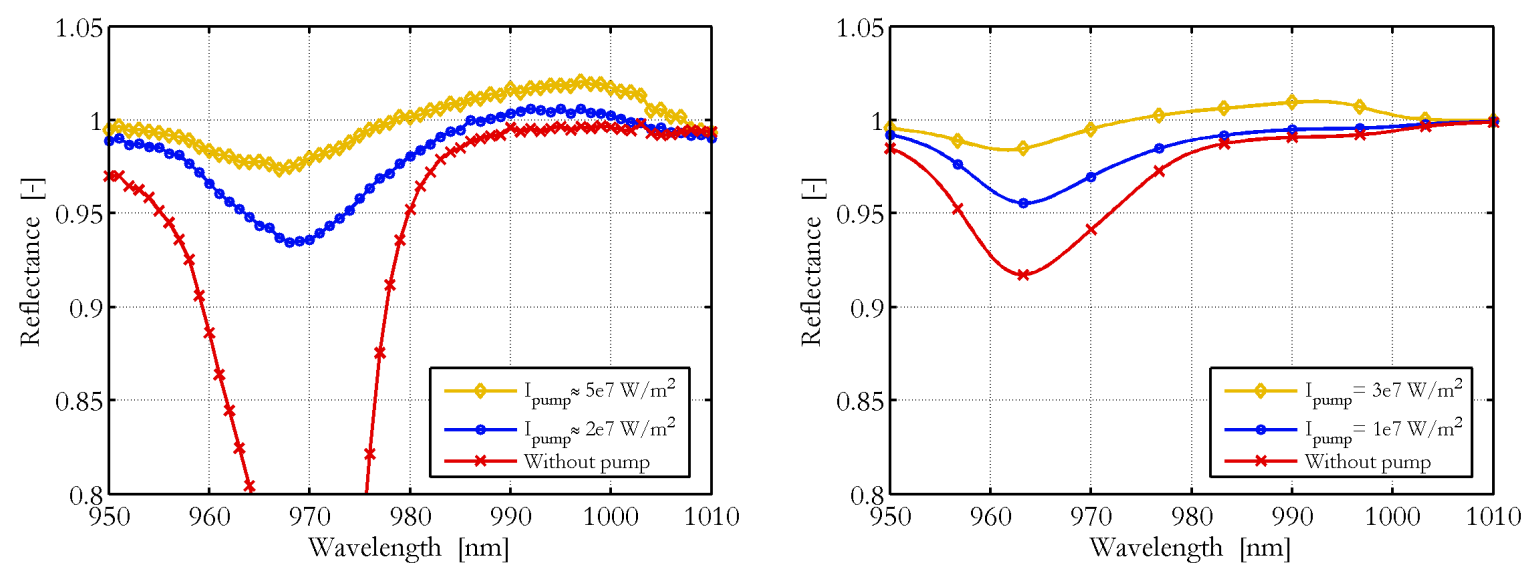

Fig. 2. Measured (left) and simulated (right) gain spectra for different absorbed pump intensities.

The authors would like to thank Hans Lindberg of Osram Opto Semiconductors for fruitful discussions.

\section{References}

[1] B. Rudin, A. Rutz, M. Hoffmann, D. J. H. C. Maas, A. Bellancourt, E. Gini, T. Südmeyer, and U. Keller, "Highly efficient optically pumped vertical-emitting semiconductor laser with more than $20 \mathrm{~W}$ average output power in a fundamental transverse mode," Opt Lett 33(22), 21792721 (2008).

[2] T. Wang, Y. Kaneda, J. M. Yarborough, J. Hader, J. V. Moloney, A. Chernikov, S. Chatterjee, S. W. Koch, B. Kunert, and W. Stolz, "HighPower Optically Pumped Semiconductor Laser at 1040 nm," IEEE Photon Technol Lett 22(9), 661-663 (2010).

[3] A. Wojcik-Jedlinska, K. Pierscinski, A. Jasik, J. Muszalski, and M. Bugajski, "Optical characterisation of vertical-external-cavity surfaceemitting lasers (VECSELs)," Optica Applicata 37(4), 449-457 (2007).

[4] J. Paajaste, S. Suomalainen, R. Koskinen, A. Härkönen, M. Guina, and M. Pessa, "High-power and broadly tunable GaSb-based optically pumped VECSELs emitting near $2 \mu \mathrm{m}$, , J Cryst Growth 311(7), 1917-1919 (2009).

[5] C. Borgentun, J. Bengtsson, A. Larsson, F. Demaria, A. Hein, and P. Unger, "Optimization of a Broadband Gain Element for a Widely Tunable High-power Semiconductor Disk Laser,” IEEE Photon Technol Lett 22(13), 978-980 (2010).

[6] A. Bellancourt, Y. Barbarin, D. J. H. C. Maas, M. Shafiei, M. Hoffmann, M. Golling, T. Südmeyer, and U. Keller, "Low saturation fluence antiresonant quantum dot SESAMs for MIXSEL integration," Opt Express 17(12), 9704-9711 (2009). 